Ann. Génét. Sél. anim., I980, 12 (I), I5-3I.

\title{
Estimation de la valeur génétique des chevaux de sport d'après les sommes gagnées dans les compétitions équestres françaises
}

\author{
B. LANGLOIS \\ avec la collaboration technique de Dominique POIREL et J.-L. BRESCH \\ Station de Génétique Quantitative et Appliquée, \\ Centre national de Recherches zootechniques, I.N.R.A., \\ 78350 Jouy-en-Josas (France)
}

\begin{abstract}
Résumé
L'exposé retrace les différentes étapes du traitement statistique des gains de chevaux dans les compétitions d'équitation française de 1972 à 1978 (dressage, saut d'obstacles et concours complets d'équitation) et aboutissant à l'estimation de la valeur génétique des reproducteurs.

Après normalisation des distributions par la transformation logarithmique, l'estimation des effets du sexe et de l'âge a été faite par la méthode des moindres carrés selon un modèle additif qui s'est avéré convenir (absence d'interactions). L'effet de l'année de la performance a été éliminé en exprimant les données corrigées pour les effets significatifs précédents en écarts réduits à une moyenne annuelle tenant compte de la proportion de non gagnants.

Les paramètres génétiques ont ensuite été estimés par décomposition de la variance selon un modèle hiérarchique père, cheval, année. Les valeurs de la répétabilité $(v)$ de la performance annuelle sont élevées pour chacune des trois disciplines et comprises entre 0,50 et 0,55 . Les valeurs de l'héritabilité $\left(h^{2}\right)$ dans les épreuves de dressage sont nulles et excluent pour l'instant toute possibilité de sélection fondée sur un tel critère. En revanche, les estimations de $h^{2}$ pour les deux autres disciplines comprises entre 0,15 et 0,20 autorisent l'établissement d'indices de sélection.

Des indices de sélection individuels utilisables pour qualifier objectivement les juments ont été établis selon la méthode classique compte tenu de $h^{2}, r$ et du nombre de performances annuelles connues.

Les indices de sélection sur descendance des étalons ont été établis selon une méthode plus originale tenant compte de l'ensemble des performances annuelles de chacun de leurs descendants. L'ensemble des indices est exprimé en unités correspondant à I / 20 d'écart-type avec une moyenne de ıoo. Chacun de ces indices est affecté d'un coefficient de détermination et par conséquent d'un intervalle de confiance.

Une vérification de l'efficacité globale des indices de sélection dont le coefficient de détermination est d'au moins 0,40 a été réalisée sur 194 couples père fils. Elle est voisine de 85 p. Ioo ce qui peut être considéré comme satisfaisant.
\end{abstract}




\section{Introduction}

Lors d'une étude préliminaire (LANGLors, I974) nous avons montré qu'après transformation logarithmique, les gains des chevaux dans les compétitions équestres en France pouvaient se prêter aux analyses statistiques et génétiques classiques et être utilisés en sélection. La prise en considération des mêmes informations au cours des années ultérieures a non seulement confirmé les premiers résultats, mais permis leur application routinière à l'estimation de la valeur génétique des reproducteurs sur la base de leurs performances individuelles (juments) ou de celles de leurs descendants (étalons).

C'est ainsi que le Service des Haras Nationaux diffusait pour la première fois au début de 1976 le classement sur descendance des étalons en "Concours de Saut d'Obstacles " (C.S.O.) et en "Concours Complets d'Équitation " (C.C.E.). Ces estimations s'enrichissant chaque année d'informations supplémentaires, trois actualisations de ces classements (étendus à celui des juments) ont été publiées depuis cette date.

Après avoir rappelé quelques résultats théoriques essentiels nous nous proposons ici de présenter les différentes étapes de l'établissement de ces indices de sélection et d'en discuter la précision et l'efficacité.

\section{I. - Matériel et méthodes}

\section{I. - Les données de base}

C'est par un système de prélèvement sur les enjeux dans les courses de chevaux que les compétitions équestres peuvent être largement dotées et qu'elles bénéficient d'une gestion moderne sur ordinateur. La "Société des Steeple-Chases de
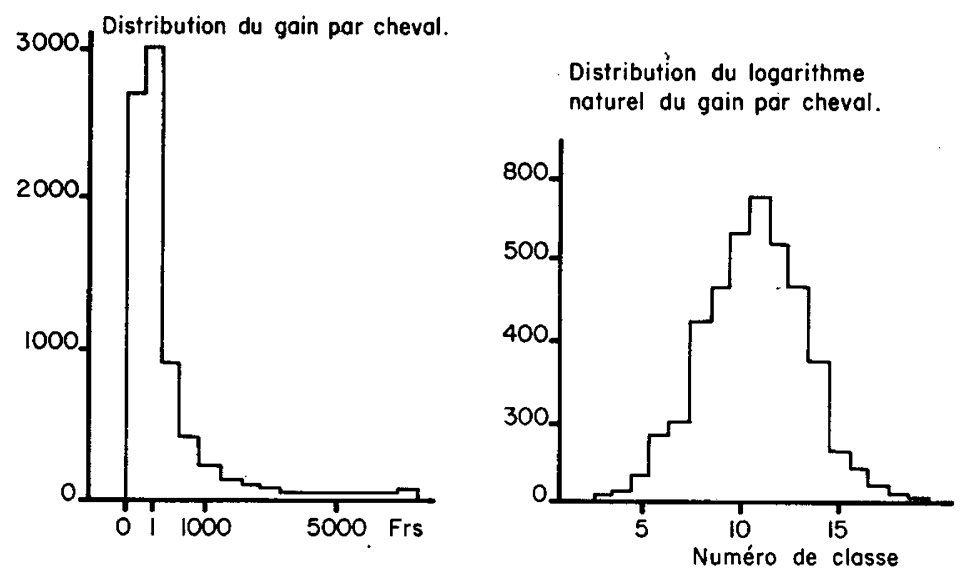

FIG. I. - Distribution de fréquence des variables dans le cas des Concours de Saut d'Obstacles en 1972.

Frequency distribution of variables for jumping shows in 1972. 
France "traditionnellement responsable des courses à obstacles assure ce travail depuis 1972. Les sommes gagnées par les chevaux dans les trois disciplines des compétitions équestres que sont par ordre d'importance les "Concours de Saut d'Obstacles " (C.S.O.), les "Concours Complets d'Équitation " (C.C.E.) et les "Concours de Dressage " (C.D.) sont ainsi publiées officiellement chaque année. Ce sont ces gains annuels que nous utilisons pour apprécier la valeur des chevaux. Données comptables ils ont l'avantage d'être recueillis et vérifiés scrupuleusement. Toutefois, de distributions extrêmement dissymétriques (fig. I), ils nécessitent une transformation pour se prêter aux traitements statistiques classiques. La transformation logarithmique s'est avérée jusqu'ici la mieux adaptée (LANGLoIs, 1974). Le Log du Gain et le Log du Gain moyen par Départ ont donc été choisis pour apprécier les performances des chevaux dans les compétitions équestres.

\section{2. - Corrections pour les effets de l'âge, du sexe et de l'année de la performance}

- Chaque année les gains nouvellement acquis par les chevaux sont soumis après transformation à une analyse de la variance selon le modèle :

$\mathrm{L}_{i j k}=\mu_{1}+a_{i}+s_{j}+r_{i j k}$

$\mathrm{L}_{i j k}=$ Logarithme naturel du gain annuel du $k^{i e m e}$ cheval de sexe $j$ et de la classe d'âge $i$

$\mu_{1} \quad$ moyenne générale

$a_{i}=$ effet de la classe d'âge $i(5$ niveaux : 4 ans, 5 ans, $6+7$ ans, $8+9+$ ro ans et Ir ans et plus)

$s_{j}=$ effet du sexe $j$ ( 2 niveaux : mâles + hongres et femelles)

$r_{i j k}=$ eflet résiduel.

- L'effet de l'année de la performance, dû aux fluctuations de la dotation, est ensuite éliminé après correction pour les effets précédents, en exprimant les variables en écarts réduits à une moyenne annuelle calculée sur l'ensemble des " partants" (" non gagnants" compris). Cela permet d'une part de tenir compte des possibles variations du pourcentage de "non gagnants" suivant les années, d'autre part d'éviter une interaction toujours possible entre l'effet de l'année et ceux de l'âge et du sexe.

\section{3. - Estimation des paramètres génétiques}

Les données ainsi corrigées année par année sont ensuite rassemblées dans un fichier général où figurent tous les résultats depuis I972. Elles ont été soumises à une analyse hiérarchique de la variance selon le modèle suivant d'après KEMPTHORNE (I957) :

$\mathrm{P}_{i j k}=\mu_{2}+\mathrm{E}_{i}+\mathrm{C}_{i j}+\mathrm{R}_{i j k}$

$\mathrm{P}_{i j k}=$ performance annuelle $k$ du cheval $j$ fils de l'étalon $i$ corrigée pour les effets de l'âge, du sexe et de l'année 
$\mu_{2}=$ moyenne générale

$\mathrm{E}_{i}=$ effet de l'étalon $i$

$\mathrm{C}_{i j}=$ effet du cheval $j$ fils de l'étalon $i$

$\mathrm{R}_{i j k}=$ effet résiduel de l'année $k$.

Ce modèle permet le calcul de l'héritabilité $h^{2}$ (4 fois la composante paternelle) et de la répétabilité $r$ (somme des composantes dues à l'étalon et au cheval).

\section{4. - Établissement des indices de sélection}

L'estimation de la valeur génétique d'un cheval ij dont on connaît $m_{j}$ performances annuelles s'écrit :

$\mathrm{I}_{i j}=\mu+\frac{m_{j} h^{2}}{\mathrm{I}+\left(m_{j}-\mathrm{I}\right) r}\left(\mathrm{P}_{i j}-\mu\right)$

$I_{i j}=$ indice génétique sur performances individuelles

$\mu \quad=$ moyenne générale

$m_{j}=$ nombre de performances annuelles de l'individu if

$h^{2}=$ héritabilité de la performance annuelle

$r \quad=$ répétabilité de la performance annuelle

$\mathbf{P}_{i j}$. = moyenne des $m j$ performances annuelles de l'individu ij

L'estimation de la valeur génétique d'un étalon $i$ d'après la moyenne $P_{i} .$. de ses $n_{i}$ descendants s'écrit :

$$
\mathrm{I}_{i}=\mu+\frac{0,5 n_{i} \lambda_{i} h^{2}}{\mathrm{I}+\left(n_{i}-\mathrm{I}\right) 0,25 \lambda_{i} h^{2}}\left(\mathrm{P}_{i . .}-\mu\right)
$$

$\mathrm{P}_{i .} .=$ moyenne des $n_{i}, \mathrm{P}_{i j}$. correspondants aux $n_{i}$ descendants de l'étalon $i$. $\lambda_{i}=$ coefficient de pondération tenant compte des déséquilibres de l'information relative à chacun des descendants de l'étalon $i$.

$$
\lambda_{i}=\frac{\frac{\mathrm{I}}{k_{i}}}{\mathrm{I}+\left(\frac{\mathrm{I}-k_{i}}{k_{i}}\right) r}
$$

Il résulte de l'expression de $\operatorname{var}\left(\mathrm{P}_{i . .}\right)$ en fonction de $\operatorname{var}\left(\mathrm{P}_{i j k}\right)$.

$r$ est la répétabilité de la performance annuelle

$k_{i}$ est la moyenne harmonique du nombre de performances $m_{j}$ de chacun des descendants de l'étalon $i$.

$$
k_{i}=\frac{\mathrm{I}}{n_{i}} \sum_{j=1}^{n_{i}} \frac{\mathrm{I}}{m_{j}}
$$

- Par ailleurs, pour plus de commodité de lecture, nous exprimons les indices en unités correspondant à $\mathrm{I} / 20$ de leur écart-type et en fixant arbitrairement 1a moyenne à la valeur Ioo. L'individu moyen vaut alors Ioo, l'individu à un écarttype au-dessus de la moyenne, I20, celui à un écart-type au-dessous, 80 . 
- La distribution des indices de sélection des étalons nous permettra d'apprécier l'efficacité de la transformation logarithmique des gains pour estimer les valeurs génétiques.

- La précision moyenne du contrôle individuel par cette méthode sera estimée d'après la distribution des coefficients de détermination des chevaux "Selle Français " nés en I968 (4 ans en I972) dont nous contrôlons toute la carrière jusqu'en I978.

- D'autre part pour les étalons des races "Selle Français » et "Anglo-Arabes » qui sont destinés à la production de chevaux de selle, l'analyse des effectifs de gagnants en fonction de la date d'entrée au haras nous fournira une estimation de la précision et de l'efficacité des compétitions équestres utilisées comme contrôle de la descendance des étalons.

\section{5. - Mesure de l'efficacité globale des indices de sélection.}

Soit $\overline{\mathrm{D}}$ la déviation moyenne des descendants d'un étalon. $2 \overline{\mathrm{D}}$ est supposé être un estimateur non biaisé de la valeur génétique $\mathrm{G}$ de cet étalon, assorti d'une erreur aléatoire $\mathrm{E}$ d'espérance nulle. On a donc :

$$
\begin{aligned}
& 2 \overline{\mathrm{D}}=\mathrm{G}+\mathrm{E}, \\
& \operatorname{Esp}(\mathrm{E})=\mathrm{O}, \\
& \operatorname{Esp}(2 \overline{\mathrm{D}})=\mathrm{G} .
\end{aligned}
$$

L'indice de sélection I est une estimation par régression de la valeur génétique $\mathrm{G}$ en son estimateur $2 \overline{\mathrm{D}}$ :

$$
\mathrm{I}=\mathrm{R}^{2} 2 \overline{\mathrm{D}}
$$

ou

$$
\mathrm{R}^{2}=\frac{\operatorname{Cov}(\mathrm{G}, 2 \overline{\mathrm{D}})}{\operatorname{Var}(2 \overline{\mathrm{D}})}=\frac{\operatorname{Var}(\mathrm{G})}{\operatorname{Var}(2 \overline{\mathrm{D}})}
$$

Les relations entre indices des étalons pères (indice $p$ ) et fils (indice $f$ ) sont donc totalement déterminées par leur coefficient de détermination $R^{2}$.

On a en effet (Mocquot, Poutous, r969) :

$\operatorname{Cov}\left(\mathrm{I}_{p}, \mathrm{I}_{f}\right)=\frac{\mathrm{I}}{2} \mathrm{R}_{p}^{2} \mathrm{R}_{f}^{2} \operatorname{var}\left(\mathrm{G}_{p}\right)$

si $\operatorname{Cov}\left(\mathrm{G}_{p}, \mathrm{E}_{f}\right), \operatorname{Cov}\left(\mathrm{G}_{f}, \mathrm{E}_{p}\right)$ et $\operatorname{Cov}\left(\mathrm{E}_{p}, \mathrm{E}_{f}\right)$ peuvent être supposées nulles

Sachant que :

$$
\operatorname{Var}\left(\mathrm{I}_{p}\right)=\mathrm{R}_{p}^{4} \operatorname{Var}\left(2 \widetilde{\mathrm{D}}_{p}\right)=\mathrm{R}_{p}^{2} \operatorname{Var}\left(\mathrm{G}_{p}\right)
$$

et

$\operatorname{Var}\left(\mathrm{I}_{f}\right)=\mathrm{R}_{f}^{q} \operatorname{Var}\left(\mathrm{G}_{f}\right)$. 
Le coefficient de régression du fils en le père s'écrira :

$b^{\mathrm{I}} f / \mathrm{I}_{p} \quad=\frac{\mathrm{I}}{2} \mathbf{R}_{f}^{2}$

si de plus on peut supposer l'absence de sélection

$\operatorname{Var}\left(\mathrm{G}_{p}\right)=\operatorname{Var}\left(\mathrm{G}_{f}\right)$

soit

$\operatorname{Var}\left(\mathrm{I}_{p}\right) \mathbf{R}_{f}^{2}=\operatorname{Var}\left(\mathrm{I}_{f}\right) \mathbf{R}_{p}^{2}$

on aura également :

$b^{\mathrm{I}} p / \mathrm{I}_{f} \quad=\frac{\mathrm{I}}{2} \mathrm{R}_{p}^{2}$

et la corrélation

$r\left(\mathrm{I}_{p}, \mathrm{I}_{f}\right)=\frac{\mathrm{I}}{2} \mathrm{R}_{p} \mathrm{R}_{f}$

La valeur de ces coefficients peut donc être calculée à la fois théoriquement et expérimentalement. Le rapport de ces deux estimations (valeur réalisée sur valeur attendue) sera qualifié " d'efficacité globale de la méthode ".

\section{II. - Résultats}

\section{I. - Effets de l'âge et du sexe}

Nous ne donnerons pas les résultats détaillés des 42 analyses qui ont été conduites ( 7 années de performances, 3 disciplines équestres et 2 variables). Ils peuvent se résumer de la façon suivante :

- dans les concours de dressage, l'effet de l'âge a été trouvé significatif cinq fois sur le Log du gain et quatre fois sur le Log du gain moyen par départ. Dans les autres cas, bien que non significative, la même tendance en faveur des chevaux les plus expérimentés pouvait être observée. De la même façon, l'effet du sexe n'est apparu significatif qu'une seule fois sur la variable Log du gain moyen par départ, mais la légère supériorité des mâles et hongres sur les femelles s'est manifestée sans aucune exception dans chacune des I4 analyses réalisées;

- dans les concours complets d'équitation, l'amélioration des performances avec l'âge est apparue significative dans toutes les analyses. L'effet du sexe en faveur des mâles et hongres rencontré de façon uniforme n'atteignait cependant pas le seuil de signification dans 5 cas sur les I4. Par ailleurs, une interaction âge-sexe significative a été rencontrée deux fois pour le Log du gain moyen par départ;

- dans les concours de saut d'obstacles, 1'effet de 1'âge a été trouvé significatif dans tous les cas. Un fort " effet 4 ans " est suivi d'une chute à 5 ans puis d'une amélioration régulière. Par ailleurs, cet " effet 4 ans " a sensiblement augmenté de I 976 à I978, à la suite de la mise en place du " cycle classique " pour les jeunes chevaux. En ce qui concerne le sexe, aucune action n'a été mise en évidence. 


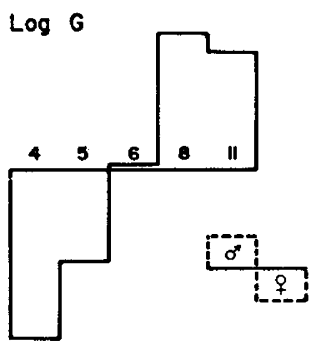

C.D.
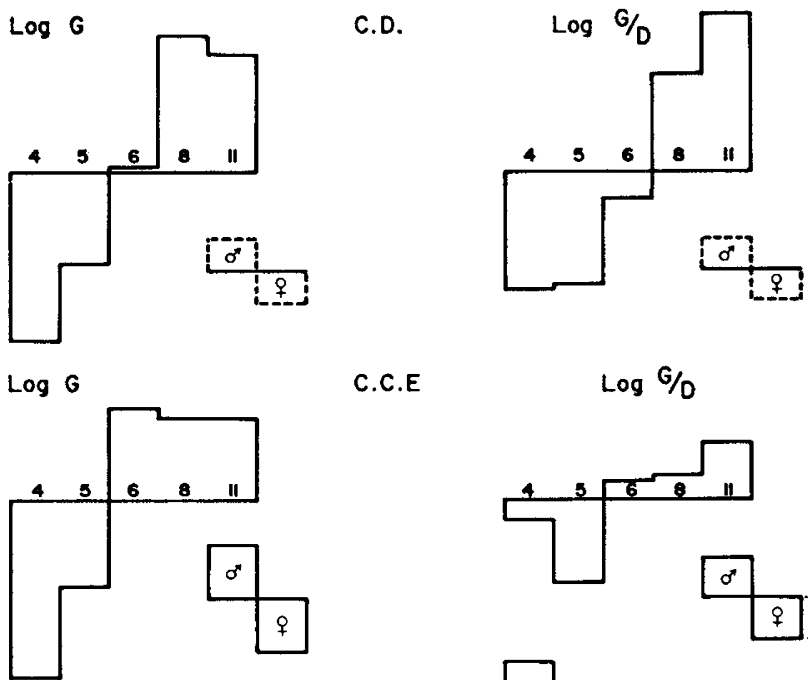

C.C.E

$$
\log 6
$$

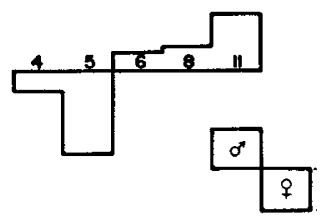

c.s.o.
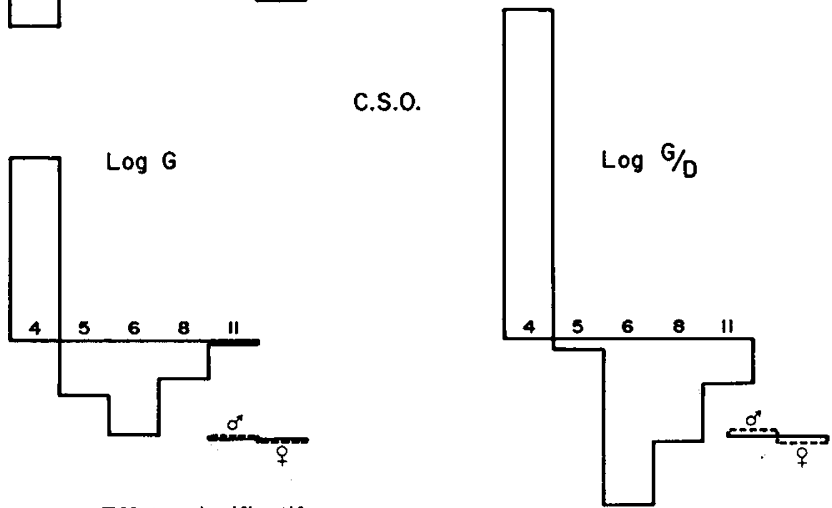

- Effets significatifs
-.-- Effets non significatifs

FIG. 2. - Schéma visualisant les effets moyens de l'age et du sexe en 1978 sur les variables Logarithme du Gain (Log G) et Logarithme du Gain moyen par Départ (Log G/D) dans les Concours de Dressage (C.D.), les Concours Complets d'Équitation (C.C.E.) et les Concours de Saut d'Obstacles (C.S.O.).

Graph showing mean effects of age and sex in 1978 on two variables Logarithm of earning (Log $G)$ and Logarithm of mean earning per stcrt (Log G/D) in dressage (C.D.), three-day events (C.C.E.) and jumping shows (C.S.O.).

La figure 2 visualise 1'ensemble des résultats pour l'année r978 et traduit assez bien ce qui a été obtenu pour les 6 années précédentes.

\section{2. - Estimation des paramètres génétiques}

Les résultats des analyses de la variance selon KEMPTHORNE (I957) sont fournis tableaux I et 2 .

Les valeurs de la répétabilité $r$ des performances annuelles d'un cheval sont élevées et comprises entre 0,46 et 0,56 pour chacune des trois disciplines étudiées. 


\section{TABLEAU I}

Résultats des analyses de la variance selon Kempthorne (1957) sur les performances de 1972 à 1974

Results of analysis of variance on performance from 1972 to 1974 according to Kempthorne (1957)

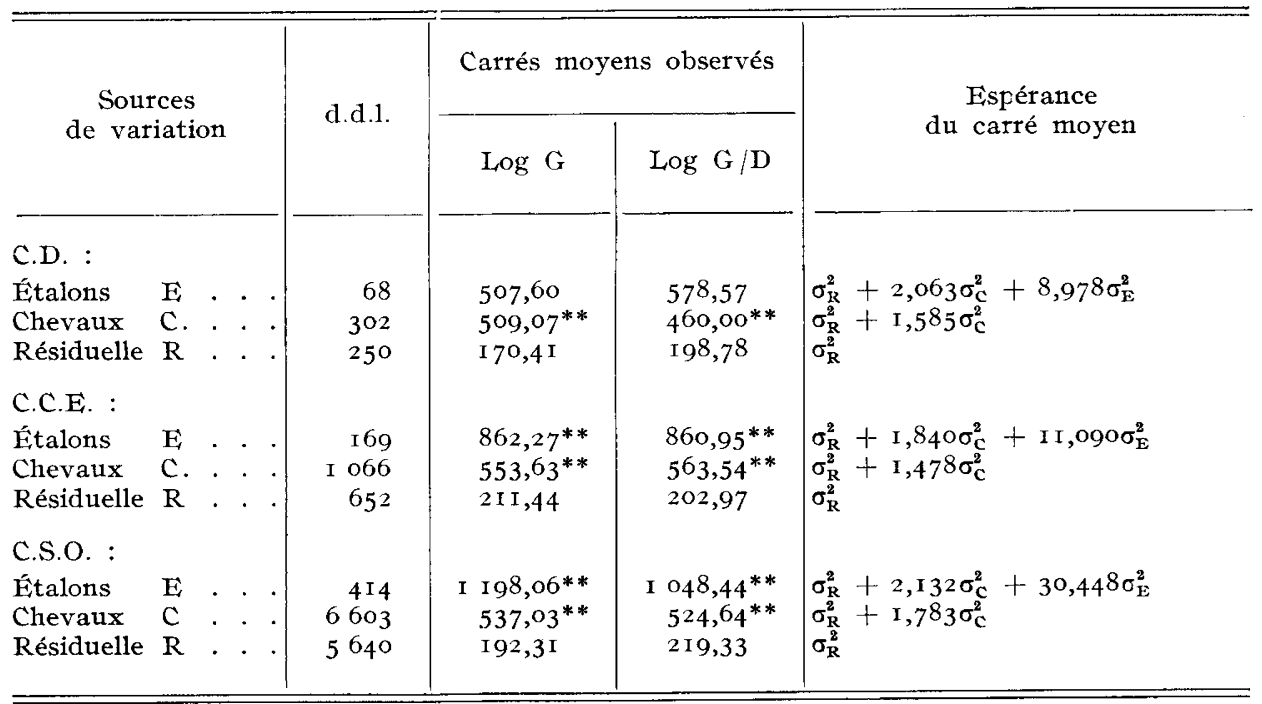

\section{TABLEAU 2}

Résultats des estimations de l'héritabilité $\left(\mathbf{h}^{2}\right)$ et de la répétabilité $(\mathbf{r})$ Results of heritability $\left(\mathrm{h}^{2}\right)$ and repeatability ( $\mathrm{r}$ ) estimations

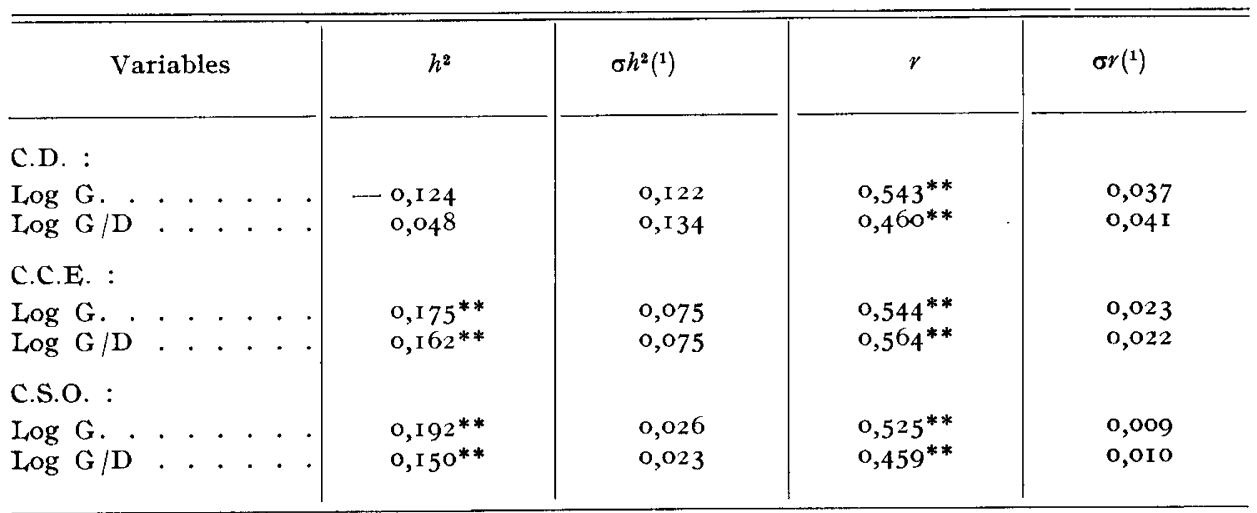
(196r).

(1) Les écart-types de l'héritabilité $h^{2}$ et de la répétabilité $r$ ont été calculés par la méthode de WooLr

$$
h^{2}=4 \frac{\sigma_{\mathrm{E}}^{2}}{\sigma_{\mathrm{E}}^{2}+\sigma_{\mathrm{C}}^{2}+\sigma_{\mathrm{R}}^{2}} \quad r=\frac{\sigma_{\mathrm{E}}^{2}+\sigma_{\mathrm{C}}^{2}}{\sigma_{\mathrm{E}}+\sigma_{\mathrm{C}}^{2}+\sigma_{\mathrm{R}}^{2}}
$$


Les estimations de 1'héritabilité $h^{2}$ dans les épreuves de dressage ne sont pas significativement différentes de zéro. En revanche, les valeurs trouvées en C.S.O. et en C.C.E. sont hautement significatives et comprises entre o,I5 et 0, I 9 .

\section{3. - Les indices de sélection}

De 1972 à 1978 , les gains de $20 \mathrm{I} 73$ chevaux ont été traités permettant 1'attribution d'un indice individuel à chacun d'entre eux et le calcul d'un indice sur descendance pour leurs 2509 pères.

Les figures 3 et 4 donnent les distributions de fréquence des indices de sélection des étalons d'après les performances de leurs descendants en C.C.E. et en C.S.O.

La figure 5 donne la distribution de fréquence des coefficients de détermination des indices individuels en C.C.E. et en C.S.O. sur la période étudiée (I972I978) pour les chevaux de Selle Français ayant eu 4 ans en I972. On peut en déduire

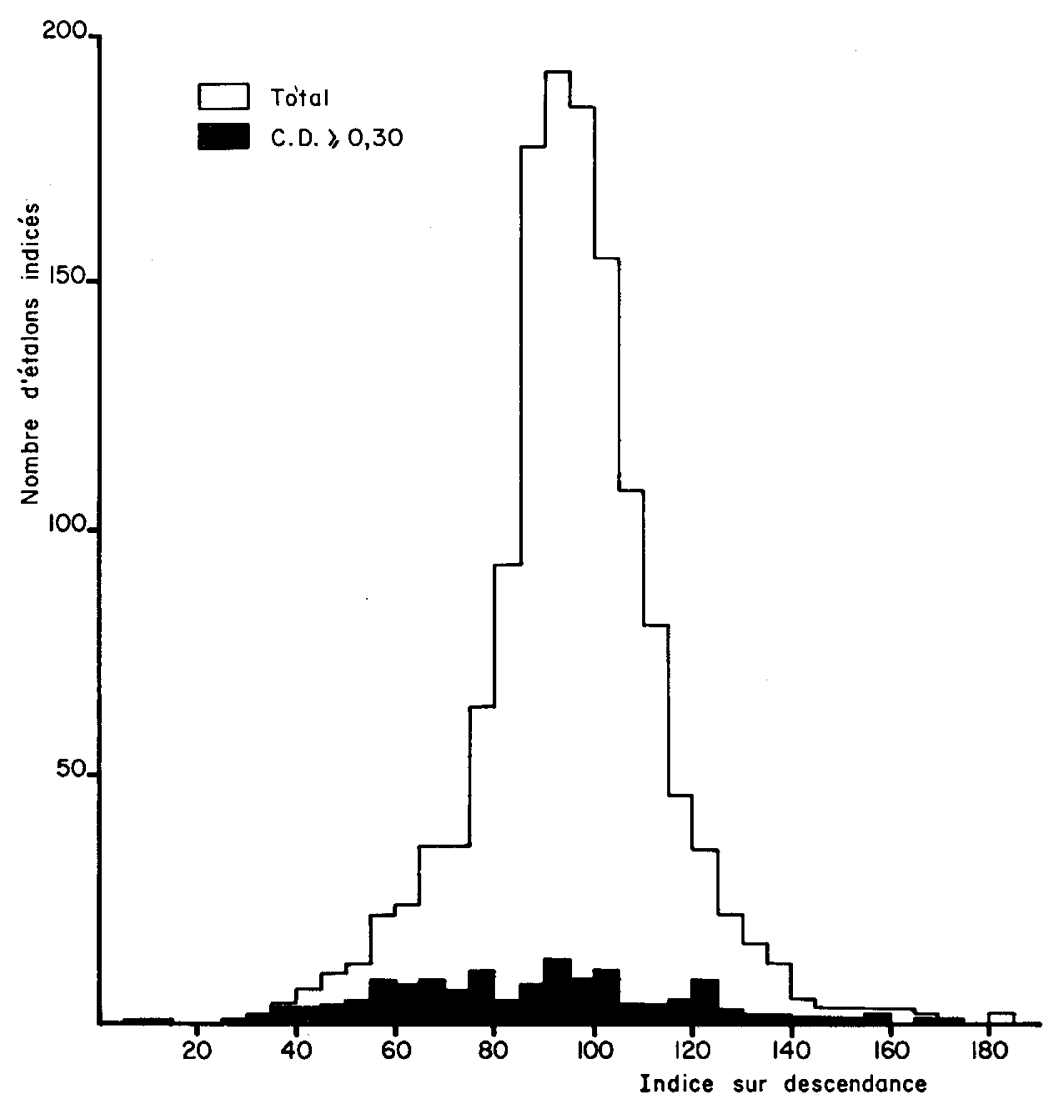

FIG. 3. - Distribution des indices sur descendance des étalons en Concours Complet d'Équitation sur la période de compétition entre 1972 et 1978.

Distribution of progeny testing indexes of stallions for three-day events during the competition period from 1972 to 1978 . 


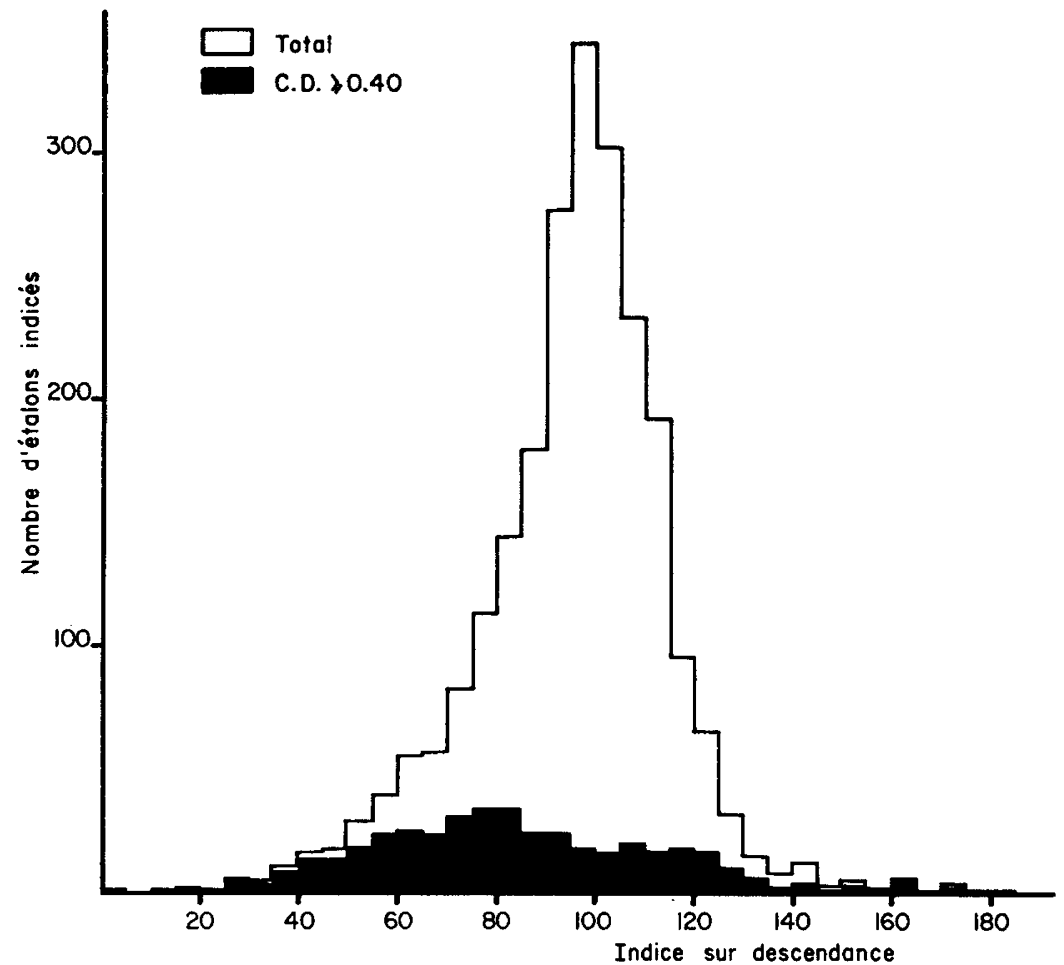

Frg. 4. - Distribution des indices sur descendance des étalons en Concours de Saut d'Obstacles sur la période de compétition entre 1972 et 1978.

Distribution of progeny testing indexes of stallions for jumping shows during the competition period from 1972 to 1978 .

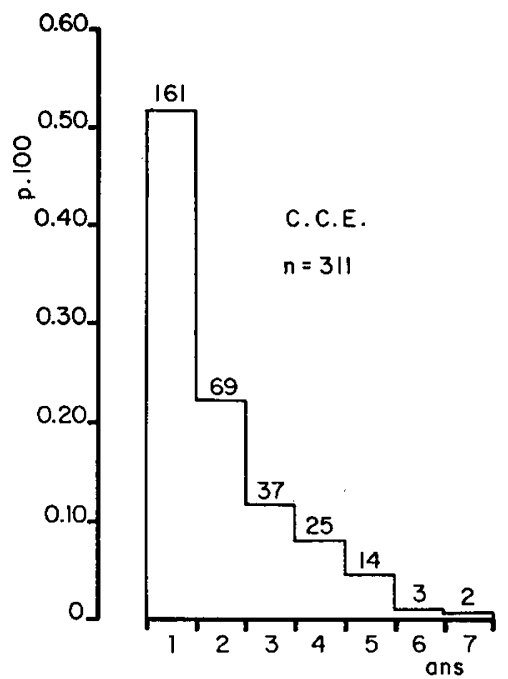

C. S.o.

$n=1122$

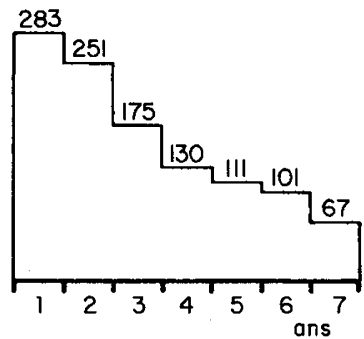

Fig. 5. - Distribution de la durée de la carrière sportive en C.C.E. et en C.S.O. d'après le nombre d'années de compétition (échantillon des chevaux de Selle Français nés en I968, les "C ", sur la période $1972-1978)$.

Distribution of career length in three day events (C.C.E.) and jumping shows (C.S.O.) according to the number of competition years (sample of "Selle Français" horses born in I968, "C", from 1972-1978). 


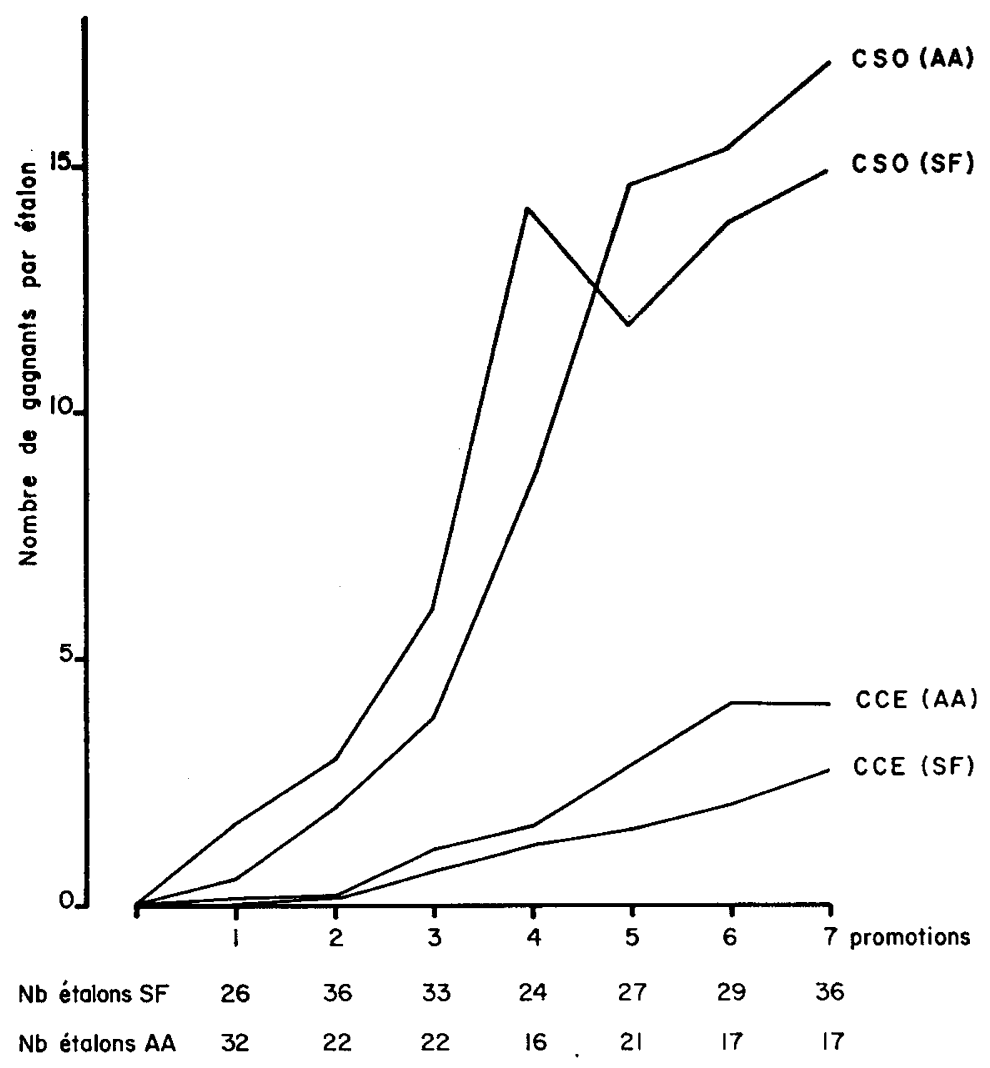

FIG. 6. - Effectifs moyens de gagnants par étalon en fonction du nombre de promotions disponibles pour participer aux compétitions.

C.C.E. = Concours Complet d'Équitation.

C.S.O. = Concours de Saut d'Obstacles.

(AA) = Étalons Anglo-Arabes.

(SF) = Étalons de Selle Français.

Mean number of winners per stallion according to the number of promotions available for competitions.

C.C.E. = Three-day event.

C.S.O. = Jumping show.

(AA) = Anglo-Arab stallions.

$(\mathrm{SF})=$ "Selle Français" stallions.

un coefficient de détermination moyen du contrôle individuel de 0,20 pour le C.C.E. et de 0,25 pour le C.S.O.

Par ailleurs, l'étude des effectifs de gagnants par étalon des races Selle Français et $A n g l o-A r a b e$ en fonction de leur date d'entrée au haras (fig. 6), permet de constater qu'en moyenne un étalon gagne de 2 à 2,5 descendants par an en C.S.O. et de $o$ à 0,5 descendant par an en C.C.F. Ils ne seront donc connus avec une précision satisfaisante (une dizaine de descendants) qu'au bout de 5 à 6 promotions en com pétitions pour le C.S.O. soit pour leur IO $^{\mathbf{e}}$ ou I $\mathbf{I}^{\mathbf{e}}$ année de monte et au bout d'une dizaine de promotions en compátition pour le C.C.E. c'est-à-dire en fait le plus souvent lorsqu'ils ont disparu. 


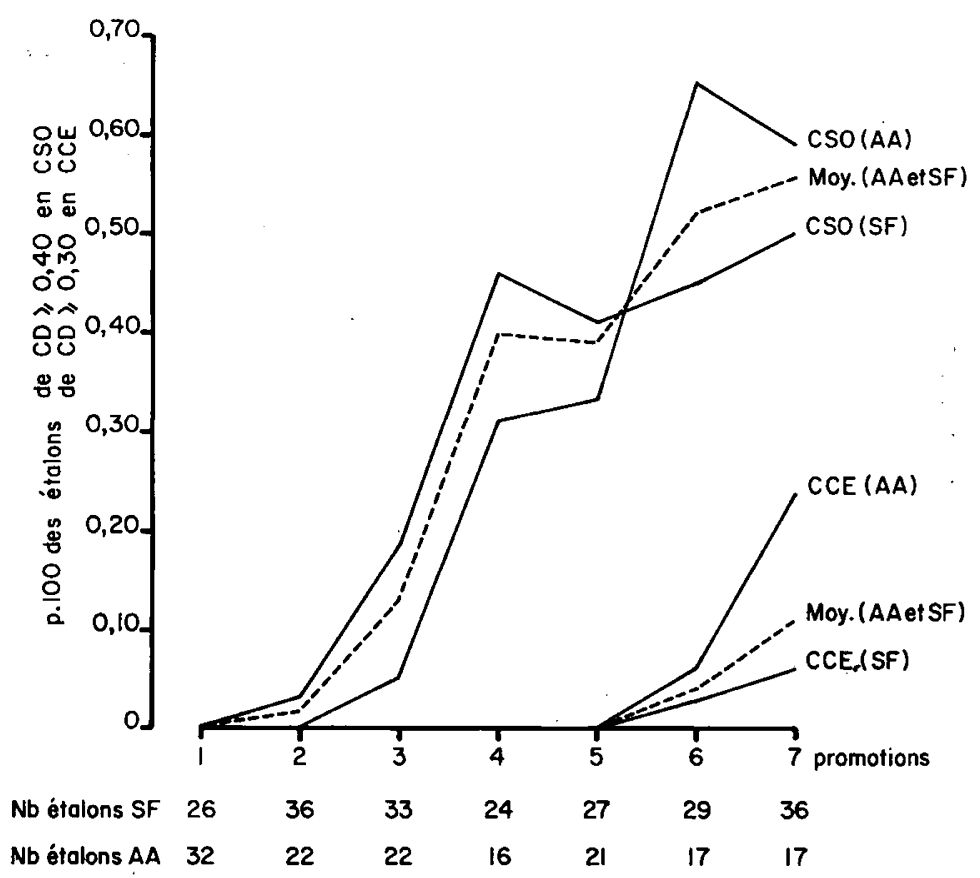

FIG. 7. - Pourcentage des étalons avec un coefficient de détermination supérieur ou égal à o,4o en C.S.O. et supérieur ou égal à o,3o en C.C.E. en fonction du nombre de promotions disponibles pour participer aux compétitions.

C.C.E. = Concours Complet d'Équitation.

C.S.O. $=$ Concours de Saut d'Obstacles.

(AA) = Etalons Anglo-Arabes.

(SF) = Étalons de Selle Français.

Percentage of stallions with a determination coefficient above or equal to 0.40 in three-day events and above or equal to 0.30 in jumping show according to the number of promotions available for competitions.

C.C.E. = Three-day event.

C.S.O. = Jumping show.

(AA) = Anglo-Arab stallions.

$(\mathrm{SF})=$ "Selle Français" stallions.

Toutefois la figure 7 qui présente le pourcentage d'étalons avec un coefficient de détermination supérieur ou égal à 0,40 en C.S.O. et supérieur ou égal à 0,30 en C.C.E. au bout de une, deux, jusqu'à sept promotions, montre qu'il existe une importante variation autour des valeurs moyennes précédemment présentées.

\section{4. - Efficacité globale}

Le tableau 3 et la figure 8 fournissent les relations théoriques et observées entre les indices sur descendance des pères et des fils pour l'aptitude au C.S.O. lorsque les $R^{2}$ sont supérieurs ou égaux à 0,40 .

Les I94 couples disponibles conduisent à une estimation de l'efficacité globale comprise entre 0,84 et 0,93 par les trois méthodes proposées. 
TABLEAU 3

Corrílation ( $\mathrm{r}$ ) et régression (b) de l'indice de sélection sur descendance en C.S.O. (I) de 194 couples d'étalons père (indice $\mathrm{p}$ ) et fils (indice f) calculés sur la période de 1972 à 1978 avec un coefficient de détermination $R^{2} \geqslant 0,40$

Correlation ( $\mathrm{r}$ ) and regression (b) of selection indexes on progeny in jumping shows. (C.S.O) (I) of 194 stallion sive ( $\mathrm{p}$ index) and -son ( $\mathrm{f}$ index) pairs calculated for the period $1972-1978$ with $R^{2} \geqslant 0,40$ as a coefficient of determination

\begin{tabular}{|c|c|c|}
\hline Valeurs observées : o & Valeurs attendues : A & Efficacité : O/A \\
\hline$b^{\mathbf{I}} / / \mathbf{1} p=0,27$ & $\frac{\mathrm{I}}{2} \overline{\mathrm{R}}_{p}^{2} \quad=0,29$ & 0,93 \\
\hline$b^{\mathbf{I}_{p / \mathbf{1}} f}=0,29$ & $\frac{\mathrm{I}}{2} \overline{\mathrm{R}}_{f}^{2} \quad=0,33$ & 0,88 \\
\hline$r\left(\dot{\mathbf{I}}_{p}, \mathbf{I}_{f}\right)=0,26$ & $\frac{\mathrm{I}}{2} \overline{\mathrm{R}}_{p} \overline{\mathrm{R}}_{f}=0,3 \mathrm{I}$ & 0,84 \\
\hline
\end{tabular}

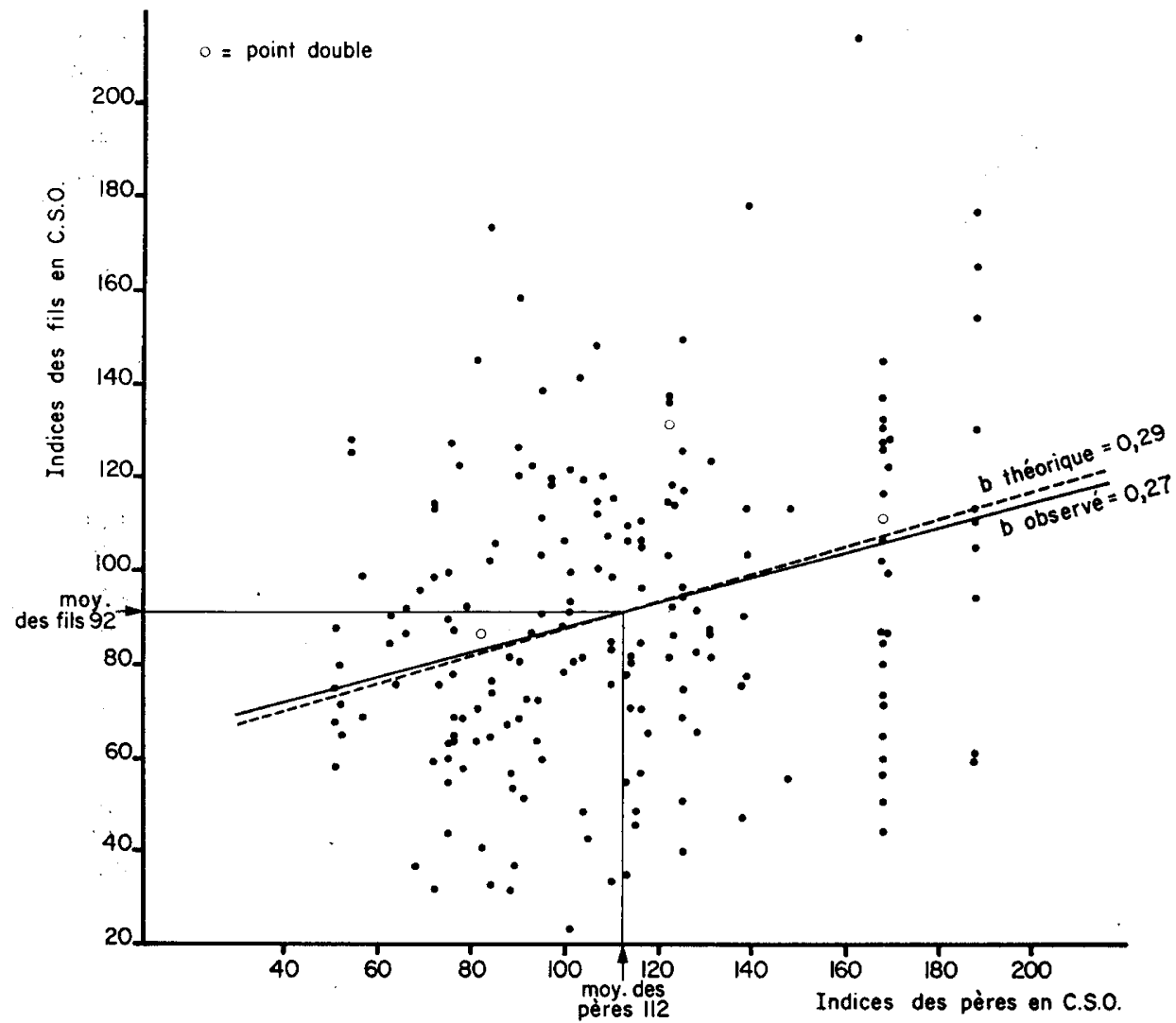

FIG. 8. - Répartition des indices des fils en fonction de l'indice de leur père et droite de régression attendue et observée. (Échantillon de I94 couples où les indices du père et du fils ont un $\mathrm{R}^{2}$ $\geqslant 0,4^{\circ}$ sur la période de compétition 1972-I978).

Distribution of indexes of sons according to sire index and regression curve expected and obtained. (Sample of 194 pairs in which the coefficient of determination indexes is $R^{2} \geqslant 0.40$ for the competition period 1972 to 1978 .) 
E.a C.C.E. la mesure de 1' " efficacité globale " n'a pas été réalisée en raison du nombre insuffisant de couples père-fils disponibles $\left(n=24\right.$ avec $\left.\mathrm{R}^{2} \geqslant 0,30\right)$.

\section{Discussion}

\section{I. - Effets de l'âge et du sexe}

L'amélioration apparente des aptitudes avec 1'âge s'est manifestée dans tous les cas (C.D., C.C.E., C.S.O.) sans que l'on puisse dire s'il s'agissait de progrès réalisés ou du résultat de la sélection progressive dont les chevaux font l'objet. L'effet " 4 ans " toujours important en C.S.O. s'est accru considérablement depuis 1975. Cette première année de compétition sert en effet de plus en plus de "banc d'essais " pour les futurs chevaux de concours hippique. Les valeurs élevées de la répétabilité semblent d'ailleurs justifier cette pratique.

Beaucoup de hongres figurant comme des mâles dans les données, l'effet de la castration n'a pas pu être estimé. La supériorité de l'ensemble "mâles et hongres " sur les femelles très apparente dans les C.C.E. est moins évidente mais régulière en dressage. La plus grande classe de galop des mâles dans le premier cas et la plus grande stabilité de tempérament des hongres dans le second pourraient peutêtre expliquer cette différence. En revanche dans les C.S.O. aucune influence du sexe n'a pu être mise en évidence. Mais dans cette discipline, le pourcentage de juments passant de 1'ordre de $40 \mathrm{p}$. Ioo à 4 ans aux environs de $20 \mathrm{p}$. foo pour les chevaux de plus de ro ans, une sélection plus intense chez les femelles que chez les mâles pourrait être invoquée.

\section{2. - Paramètres génétiques}

Les valeurs de répétabilité des performances annuelles d'un cheval sont élevées et voisines de 0,50 pour chacune des trois disciplines étudiées. Il en résulte que l'information apportée par une année de performances est déjà bien représentative de la valeur d'un animal et que deux ou trois années suffisent pour se faire une idée convenable de chaque individu.

L'estimation de l'héritabilité dans les épreuves de dressage n'est pas signicativement différente de zéro et écarte pour l'instant toute possibilité de sélection fondée sur l'information apportée par les gains. En effet l'influence du cavalier est sans doute prépondérante dans cette discipline et les différences entre chevaux exprimées par ce critère sont plus le résultat de variations dans la quantité et la qualité du travail accompli par les dresseurs que de différences d'aptitude entre chevaux.

En revanche, les valeurs hautement significatives trouvées en C.C.E. et en C.S.O. comprises entre $0, I_{5}$ et 0,20 autorisent l'estimation de la valeur génétique des reproducteurs. La prise en considération du nombre de départs sans influence apparente en C.C.E. semble toutefois diminuer la qualité de l'information en C.S.O., les valeurs de l'héritabilité et de la répétabilité étant alors sensiblement plus faibles. Ceci provient sans doute du fait qu'en C.S.O. la participation d'un cheval à une épreuve ne vise pas toujours à ce qu'il se classe mais souvent aussi à son dressage ou à l'apprentissage d'un cavalier. 
Il résulte de ces considérations que seule la variable logarithmique du gain a été utilisée pour l'estimation de la valeur génétique des reproducteurs.

\section{3. - Les indices des sélection}

Bien qu'il soit calculé sur l'ensemble des chevaux de compétition (mâles, femelles et hongres), l'indice de sélection individuel peut servir directement de base objective pour la qualification des juments au sein d'un "livre d'élite " pour chaque spécialité (C.S.O. et C.C.E.). En effet, 2 fois plus précis que la qualification traditionnelle sur " modèle et allures " (LANGLors et al., I978), il devrait progressivement la remplacer dans la mesure où l'on s'oriente vers la création de lignées spécialisées pour la haute compétition (LEGAUL, I975).

Plus précis, les indices de sélection sur descendance ne sont cependant disponibles que lorsque les étalons sont âgés. Il apparaît en effet que les compétitions ne concernent qu'une faible fraction de la production des étalons, celle qui se trouve entre les mains des cavaliers peu nombreux qui la pratiquent. De ce fait, le contrôle de la descendance des étalons est extrêmement lent et contraint dans les conditions actuelles à ne pratiquer la sélection que sur ascendance ou au mieux sur performances individuelles.

L'utilisation de l'insémination artificielle permettrait de rendre le test de la descendance plus rapide et de mieux valoriser les meilleurs pères ainsi confirmés. De même la création d'une lignée spécialisée à partir des juments contrôlées sur performances et des étalons confirmés sur descendance permettrait sans doute d'éviter la dispersion des moyens de contrôle sur un trop grand nombre de reproducteurs, ce qui est une des principales raisons de la lenteur du test.

En effet la demande des cavaliers de compétition se trouvant mieux canalisée sur la production d'un nombre plus restreint d'étalons, on en connaîtrait beaucoup plus rapidement la valeur.

\section{4. - Efficacité globale}

On peut remarquer de prime abord que la sélection sur l'aptitude au saut ne semble pas très intense puisque la valeur moyenne des pères considérés n'est que de II2. La variabilité génétique est donc probablement équivalente chez les pères et les fils. L'utilisation des trois modes d'estimation de l'efficacité globale de la méthode d'indexation paraît donc justifiée. Les résultats obtenus par la corrélation et les deux régressions $0,84^{-0,88}$ et 0,93 sont assez élevés et confirment une bonne fiabilité générale de ces indices de sélection qui en l'absence actuelle de toute autre information rationnelle doivent guider les éleveurs pour apprécier les performances des chevaux.

\section{Conclusion}

Les gains dans les compétitions équestres peuvent servir à l'amélioration génétique de l'aptitude au saut d'obstacles. Toutefois, étant donné la longueur de l'intervalle de génération dans les populations chevalines (Io à I2 ans), la faible précision moyenne du contrôle individuel $\left(h^{2}=0,20\right)$ et la lenteur du contrôle 
de la descendance, la mise en place d'une lignée spécialisée pour le C.S.O. semble devoir s'imposer si l'on veut véritablement créer un matériel génétique hautement performant pour cette discipline. Dans cette orientation l'utilisation des techniques modernes de reproduction apparaît comme une nécessité impérative. Sur le plan économique cette lignée trouvera un débouché auprès des cavaliers de compétition et sur un marché international en pleine expansion. Elle pourra ensuite jouer le rôle de réservoir d'aptitude au saut pour d'autres orientations de l'élevage pour lesquelles cette qualité n'est qu'un élément de l'objectif poursuivi.

Dans une optique plus traditionnelle, les indices de sélection proposés doivent servir de référence pour apprécier les performances dans la sélection. Ils représentent en effet, malgré leurs faibles précisions, un progrès considérable sur les usages antérieurs qui ne considéraient que le gain brut.

Rę̧u pour publication en Mai 1980

\title{
Remerciements
}

Ce travail a été réalisé dans le cadre de la convention passée entre l'administration des Haras et 1'Institut National de la Recherche Agronomique. Il n'aurait pu être conduit saris la participation du Service des compétitions équestres de la Société des Steeple-Chases de France que nous remercions de l'établissement et de la bonne gestion des fichiers de base.

Nous sommes en fin profondément reconnaissants à Monsieur LEGAUL, T, maître de recherches à l'I.N.R.A., qui fut l'initiateur de ces études auxquelles il n'a jamais cessé d'apporter son soutien et sa réflexion.

\section{Summary}

\author{
Estimation of the breeding value of the sport horses \\ from their earnings in the french shows
}

The different steps of the statistical analysis of horse earnings from 1972 to 1978 in French shows (dressage, jumping and "three days event") for estimating the breeding value of animals are described.

After logarithmic transformation and adjustment for "sex" and "age" effects estimated by the least squares method (in case of significance), data are expressed as standardized deviations from year average.

Genetic parameters were estimated through components of variance analysed on a hierarchical model. Repeatability estimates $(r)$ were high and ranged between. 50 and .55. Heritability estimates $\left(h^{2}\right)$ for "dressage" are not significantly different from zero; on the other hand, heritability values ranged between . I5 and .20 for the two other trials and allowed establishment of selection index.

Performance testing indexes for qualifying breeding mares are built on the classical method, taking account of $h^{2}, r$ and of the number of yearly performances of each horse.

Progeny testing indexes of stallions take account of all yearly performances of each offspring. All selection index are expressed in units cotresponding to $I / 20$ standard deviation around the general mean of roo. Each index is associated with a "coefficient of determination" and consequently with a "confidence intervall".

The global efficiency of progeny testing indexes has been measured on 194 father-son pairs whose coefficient of determination was $\geqslant .4^{\circ}$. This efficiency can be considered as satisfactory since it approaches 85 per cent. 


\section{Références bibliographiques}

BURdetTe W. J., I963. Methodology in mammalian. Genetics, Holden Day, San Francisco. KEMPTHORNE O., 1957. An introduction to genetic statistics. John Wiley and Sons. New York. LANGLoIs B., I974. Résultats de recherches en matière génétique chez le cheval. II. Étude des gains des chevaux dans les Concours de Saut d'Obstacles (résultats préliminaires). Journ. ét. comm. chev. Fed. eurp. zootech., Copenhague, I7-2I août 1974, 14 p. et Livest. Prod. Sci., 2, I9I-204.

Langlois B., Froidevaux J., Lamarche L., Legault C., Iegault P., Tassencourt L., Thíret M., I978. Analyse des liaisons entre la morphologie et l'aptitude au galop, au trot et au saut d'obstacles chez le cheval. Ann. Génét. Sél. anim., I978, 10, (3), 443-474.

LEGAULT C., r975. Can we select systematically for jumping ability ? Proceeding of the international Symposium on Genetics and Horse Breeding, I7-I9 septembre I975. Royal Dublin Society. P. 7 I-77.

Mocguot J. C., Poutous M., I969. Relations entre les index laitiers des reproducteurs mâles pères et fils de race bovine Française Frisonne, Pie noire. Ann. Génét. Sél. anim., 1, (3) 273-28o.

Woolf B., i96r. (Cité d'après FAlconer p. 209 dans BurdetTe i963). 